\title{
Revisão sistemática sobre alotransplantes de ilhotas de Langerhans em roedores: análise de sítio de transplante e tempo de sobrevida
}

\author{
Systematic review of islet allotransplantation in rodents: analysis of \\ transplantation site and survival time
}

\author{
Leandro Ryuchi Iuamoto ${ }^{1}$, Alberto Meyer ${ }^{1}$, Eleazar Chaib ${ }^{1}$, \\ Luiz Augusto Carneiro D'Albuquerque ${ }^{1}$
}

Iuamoto LR, Meyer A, Chaib E, D’Albuquerque LAC. Revisão sistemática sobre alotransplantes de ilhotas de Langerhans em roedores: análise de sítio de transplante e tempo de sobrevida / Systematic review of islet allotransplantation in rodents: analysis of transplantation site and survival time. Rev Med (São Paulo). 2014 out.-dez.;93(4):151-8.

RESUMO: Introdução. A escassez de ilhotas é uma restrição importante ao uso de ilhotas pancreáticas para o desenvolvimento do alotransplante para o tratamento do diabetes tipo I. É necessário, portanto, desenvolver fonte ilimitada de células produtoras de insulina. Objetivos. Organizar e analisar dados sobre o sítio de transplante e o tempo de sobrevida relacionados a tentativas experimentais de alotransplante em roedores, a fim de permitir elaborar um modelo mais adequado para suprir a escassez de doadores de ilhotas. Metodologia. Realizamos uma revisão sistemática usando o banco de dados Pubmed para pesquisar artigos publicados que contenham as palavraschaves "rodent islet transplantation". Foram incluídos estudos envolvendo experimentos alotransplante de ilhotas de roedores e analisadas as listas de referências das publicações recuperadas. Artigos relacionados a isotransplantes, autotransplantes e xenotransplantes foram excluídos do estudo. Resultados. Um total de 30 estudos relacionados a alotransplantes em diferentes sítios de enxerto foram selecionados para a revisão sistemática baseados na relevância de seus dados e em sua atualização. O fígado e a cápsula renal são sítios que possibilitaram uma maior sobrevida das ilhotas transplantadas. Conclusão. O alotransplante em roedores é promissor e continua a se desenvolver em diversos centros. As taxas de sobrevivência de aloenxertos aumentaram com a utilização de diferentes medicamentos e locais de enxerto.

DESCRITORES: Transplante de ilhotas pancreáticas; Hiperglicemia; Modelo animal; Aloenxertos; Imunossupressão; Literatura de revisão como assunto.

\begin{abstract}
Introduction. The scarcity of islets is an important use of pancreatic islets for the development of allograft for the treatment of diabetes type I. It is therefore necessary to develop unlimited source of insulin-producing cells restriction. Objectives. Organize and analyze parameters related to the site of transplantation and survival time related to experimental rodent allotransplantation attempts in order to allow the elaboration of the most suitable model to supply the scarcity of islet donors. Methodology. We performed a systematic review using the PubMed database to search published articles containing the keywords "rodent islet transplantation". We included studies involving allotransplantation experiments with rodents' islets and we reviewed the reference lists of the publications retrieved that were eligible. We excluded articles related to isotransplantation, autotransplantation and xenotransplantation such as transplantation in other species. Articles related to isotransplantations, transplantations and xenografts were excluded from the study. Results. A total of 30 related allografts in different sites of graft studies were selected for the systematic review based on their relevance data and update. The liver and the kidney capsule are sites that showed better survival of transplanted islets. Conclusion. Allograft transplantation in rodents is promising and continues to develop in several centers. The allograft survival rates increased with the use of different drugs and graft sites.
\end{abstract}

KEYWORDS: Islets of Langerhans transplantation; Hyperglycemia; Animal model; Allografts; Immunosuppression; Review literature as topic.

1.Unidade de Transplante e Cirurgia do Fígado e Pâncreas, Departamento de Gastroenterologia Faculdade de Medicina da Universidade de São Paulo, São Paulo, Brasil.

Endereço para correspondência: Unidade de Transplante e Cirurgia do Fígado e Pâncreas, Departamento de Gastroenterologia Faculdade de Medicina da Universidade de São Paulo, São Paulo, Brasil, Av. Dr. Enéas de Carvalho Aguiar 255 - $9^{\circ}$ andar. São Paulo, SP, Brasil. CEP: 05403-090. E-mail: leandro.iuamoto@gmail.com 


\section{INTRODUÇÃO}

Em crianças e adolescentes, a incidência de diabetes tipo 1 aumentou $4 \%$ no último ano. Uma das tendências atuais é a da ocorrência do diabetes mellitus tipo $1 \mathrm{em}$ faixas de idade cada vez mais baixas, principalmente entre 10 e 14 anos $^{1}$.

Na população mundial, estima-se que $4 \%$ seja portadora de diabetes mellitus, dentre os quais $10 \%$ possuem diabetes tipo $1^{2}$. Hoje, a doença já atinge de 0 a 5 anos $^{2}$. Segundo o CENSO-IBGE-2010, atualmente há 12.054.827 de diabéticos no Brasil. Tendo em vista o contexto da doença atual, aproximadamente, 1,2 milhões de diabéticos no Brasil podem ser beneficiados com linhas de pesquisa relacionadas ao tratamento de diabetes tipo $1^{3}$.

Até hoje, a insulinoterapia constitui o principal método de tratamento da doença, entretanto, cerca de 5\% a $10 \%$ dos pacientes apresentam flutuações intensas e inesperadas das suas glicemias, resultando em múltiplos episódios de hipoglicemia com repercussões clínicas graves. Nesses casos, o transplante de pâncreas seria a alternativa que já está em uso clínico e o transplante de ilhotas de Langerhans é a alternativa menos invasiva em desenvolvimento. Em relação a eficácia do tratamento, alguns resultados mostraram $70 \%$ de insulinoindependência no primeiro ano de pós-operatório de pacientes tratados com o transplante de ilhotas, porém, a taxa de sobrevida das ilhotas permanece baixa ${ }^{4}$.

A escassez de ilhotas é um obstáculo significativo ao uso generalizado de ilhotas para o alotransplante. De acordo com a Rede de Procura de Órgãos e Transplantes, em 2011, apenas 1562 pâncreas foram recuperados de 8.000 doadores de órgãos disponíveis nos Estados Unidos. Além de muitos doadores de pâncreas não se adequarem a extração de ilhotas, por não satisfazerem os critérios de seleção e o fato da inadequada manipulação das ilhotas, apenas um pequeno número de transplantes de ilhotas pode ser efetuado 5 .

Algumas restrições foram encontradas no avanço das técnicas do transplante de ilhotas: o fato de o número de doadores de pâncreas para o transplante de ilhotas estar aquém do necessário para a cura de milhões de indivíduos portadores de diabetes tipo 1; as dificuldades técnicas e o custo do isolamento das ilhotas; pouca durabilidade da insulinoindependência; além da autoimunidade e a rejeição pós-transplante que ainda devem ser superadas. É fundamental, portanto, que se desenvolva uma fonte ilimitada de células capazes de secretar insulina em resposta à glicose e passíveis de serem transplantadas com pouca ou sem a necessidade de imunossupressão sistêmica ${ }^{6}$.

A técnica de transplante de ilhotas vem sendo desenvolvida para se adquirir uma fonte adequada de insulina no transplantado, solucionando o problema da escassez de doadores.

Os primeiros sucessos em aloenxertos de ilhotas no tratamento cirúrgico de diabetes ocorreram em $1990 \mathrm{com}$ Scharp et al. ${ }^{4}$ que alcançaram a insulino-independência em um paciente portador de DM1 em um intervalo de um mês. No entanto, muitas dificuldades técnicas foram encontradas para prosseguir no desenvolvimento da reprodução deste experimento. Na década de 1991 a 2000, foram realizadas 450 tentativas de transplante de ilhotas em pacientes com DM1, com índice de sucesso de apenas $8 \% .50 \%$ do sucesso dos casos foram obtidos e reportados, quando os pacientes tinham se tornado diabéticos por terem sido submetidos à pancreatectomia ${ }^{4}$.

Até que em 1999/2000, Shapiro et al. obtiveram sucesso na independência de insulina em 7 pacientes diabéticos, realizando os experimentos com base no protocolo modificado de Edmonton?

O transplante de ilhotas cada vez mais tem mostrado uma intervenção que apresenta morbidade 20 vezes menor que o transplante de pâncreas, por ser muito menos invasivo do ponto de vista cirúrgico ${ }^{8}$.

\section{OBJETIVOS}

O objetivo do estudo é realizar a revisão das tentativas experimentais de alotransplantes em roedores, de modo a analisar os aspectos envolvidos no sítio anatômico de transplante e o tempo de sobrevida dos roedores transplantados.

\section{MÉTODOS}

O trabalho foi realizado utilizando a base de dados Pubmed procurando por artigos publicados contendo as palavras-chaves: "rodent islet transplantation".

Foram encontrados 2650 artigos de 2000 a 2013, mas somente 30 artigos referentes a alotransplante foram selecionados com base em sua relevância e na atualização de seus dados.

\section{Processo de pesquisa}

Utilizamos o banco de dados Pubmed para pesquisar artigos publicados que contivessem as palavraschave: "rodent islet transplantation". Entretanto, a fim de filtrar os resultados, procuramos apenas registros do PubMed do período que engloba janeiro de 2000 a dezembro de 2013 utilizando os seguintes termos de pesquisa relacionados a alotransplante de ilhotas 
em roedores: ((()((rodent islet transplantation) AND (“2000”[Date - Completion]: “3000”[Date - Completion])) AND allotransplantation)) NOT porcine) NOT tilapia) NOT nonhuman primate.

Assim, artigos envolvendo estudos em suínos, tilápia e primatas não-humanos (espécies mais comuns utilizadas para o alotransplante de ilhotas) foram excluídos de nossa análise, a fim de filtrar apenas trabalhos relacionados ao alotransplante em roedores. Em seguida, revisamos as listas de referências das publicações recuperadas e obtivemos textos completos de publicações que potencialmente poderiam ser incluídas na revisão sistemática. Estudos não publicados e cartas foram ignorados. Artigos que não têm texto completo em Inglês disponíveis foram comprados para revisão.

Trabalhos que foram considerados potencialmente elegíveis foram selecionados para análise com os seguintes critérios de inclusão:

$\checkmark$ Estudo deve estar relacionado ao alotransplante de ilhotas;

$\checkmark$ As espécies estudadas devem ser obrigatoriamente espécies de ratos;

$\checkmark$ Relevância e atualização de informações dos artigos.

Informações relevantes foram dispostas em tabelas, tais como doador/receptor; imunossupressão; local do aloenxerto e tempo de sobrevivência do enxerto.

\section{RESULTADOS}

Um total de 2.650 artigos de 2000 a 2013 foram encontrados, mas apenas 30 artigos foram relacionados a alotransplante. Estes artigos foram selecionados com base em sua relevância e atualização dos dados.

\section{DISCUSSÃO}

No presente estudo, foram revisados artigos científicos cujos experimentos consistiram em utilizar roedores semelhantes na idade e no peso para serem submetidos ao alotransplante.

É importante notar que o desenvolvimento de imunossupressores possibilitou uma maior taxa de sobrevida nos experimentos envolvendo alotransplante de ilhotas em ratos. A variedade na utilização de sítios de transplantes colaborou com uma melhor sobrevida dos roedores transplantados, devido a peculiaridades como vascularização, localização no organismo e reatividade imune.

Em relação aos diferentes sítios para transplantes estudados, de acordo com a tabela, a cápsula renal foi o sítio mais utilizado para transplante. Em segundo lugar, temos o fígado - veia porta ${ }^{16,19}$, o baço ${ }^{9,22}$, o sítio intraperitoneal ${ }^{13}$, espaços subconjuntival e subretinal ${ }^{14}$, medula óssea (tibia) ${ }^{15}$ e prega cutânea dorsal - câmara não-metálica ${ }^{11}$, subcutâneo ${ }^{26}$, foram sítios utilizados em pequena porcentagem.

Wee et al. ${ }^{14}$ utilizou a veia porta (fígado) como sítio de alotransplante e sacrificou as cobaias com 100 dias de pós-operatório; Melzi et al. ${ }^{23}$, Watanabe et al. ${ }^{16}$ e Merani et al. ${ }^{10}$ obtiveram uma taxa de sobrevida de até 100 dias, onde o sítio de recepção do alotransplante foi a cápsula renal.

É importante notar que Merani et al. ${ }^{10}$ utilizaram AEB-071 (Proteína Cinase C inibitória) + CsA, CTLA4Ig, MMF em seus grupos de estudo, enquanto Watanabe et al. ${ }^{16}$ realizaram experimentos com Tacrolimus e DHMEQ (inibidor de NF- kB). Melzi et al. ${ }^{23}$ utilizaram Rapamicina+ FK506+ anti-IL-2Ra, mAbs e rapamicina+IL-10 em seus experimentos. Assim, o efeito do imunossupressor pode ter exercido grandes influências na rejeição do enxerto de ilhotas pancreáticas, colaborando com uma maior taxa de sobrevida em relação aos demais resultados obtidos por outros autores.

\section{CONCLUSÃO}

A partir dos estudos analisados, podemos inferir que o aloenxerto em roedores é promissor e continua em desenvolvimento. As taxas de sobrevida vêm aumentando com a utilização de diferentes sítios para enxerto. Este fato pode permitir a superação do problema da escassez de doadores de ilhotas e possibilidades dos avanços no tratamento do diabetes tipo 1 .

Com base nos estudos analisados, pode-se inferir que as taxas de sobrevivência de aloenxertos aumentaram com a utilização de diferentes locais de enxerto. O sítio de transplante que resultou em maiores taxas de sobrevida foi a cápsula renal.

Tabela 1: Os sítios de enxerto de ilhotas pancreáticas com base na literatura da base de dados PubMed

\begin{tabular}{c|c}
\hline $\begin{array}{l}\text { Sítios de enxerto de } \\
\text { ilhotas }\end{array}$ & $\begin{array}{l}\text { Literatura da base de dados } \\
\text { PubMed }\end{array}$ \\
\hline Cápsula renal & $70 \%$ \\
\hline Fígado & $23 \%$ \\
\hline Outros sítios* & $7 \%$ \\
\hline
\end{tabular}

* Subcutâneo, medula óssea, espaço sub-retinal, espaço subconjuntival, baço, cavidade peritoneal e espaço da submucosa do duodeno. 
Iuamoto LR, et al. Revisão sistemática sobre alotransplantes de ilhotas de Langerhans em roedores.

Tabela 2: Descrição dos estudos experimentais realizados em alotransplantes em roedores

\begin{tabular}{|c|c|c|c|c|}
\hline Autores & Doador/Receptor & Imunossupressão & Sítio de alotransplante & Tempo de sobrevida do enxerto \\
\hline Fotiadis et al. ${ }^{9}$ & Lewis $\rightarrow$ Wistar & $\begin{array}{l}\text { Micofenolato mofetil } \\
(\mathrm{MMF}) \text { and Ciclosporina A (CsA) }\end{array}$ & Baço & $\mathrm{N} / \mathrm{A}$ \\
\hline Merani et al. ${ }^{10}$ & Lewis $\rightarrow$ Wistar & $\begin{array}{l}\text { AEB-071 (Proteína Cinase C inibitória) } \\
+ \text { CsA, CTLA4-Ig, MMF }\end{array}$ & Cápsula renal & 100 dias \\
\hline Nishimura et al. ${ }^{11}$ & $\mathrm{C} 57 \mathrm{BL} / 6 \rightarrow \mathrm{Balb} / \mathrm{c}$ & Tacrolimus & $\begin{array}{l}\text { Câmara de dobras cutâneas } \\
\text { dorsal não metálica }\end{array}$ & $\mathrm{N} / \mathrm{A}$ \\
\hline Makhlouf et al. ${ }^{12}$ & $\mathrm{C} 57 \mathrm{BL} / 6 / \mathrm{Balb} / \mathrm{c}$ & $\begin{array}{l}\text { Bloqueio de CD28:B7 e anti-CD40L; } \\
\text { CTLA-4 }\end{array}$ & Cápsula renal & 1 semana \\
\hline $\begin{array}{l}\text { Salazar-Bañuelos } \\
\text { et al. }{ }^{13}\end{array}$ & $\begin{array}{l}\text { Wistar } \rightarrow \text { Sprague } \\
\text { Dawley }\end{array}$ & Sem imunossupressão & Canal medular & 21 dias \\
\hline Wee et al. ${ }^{14}$ & Lewis $\rightarrow$ Fisher & CsA + Tautomycetin $(\mathrm{TMC}=$ synergist $)$ & Fígado (veia porta) & $\begin{array}{l}\text { Grupo controle }-5.2 \text { dias }( \pm 0.5) \\
\text { TMC }-5,1 \text { dias }( \pm 0,9) \\
\text { TMC }(0.03 \mathrm{mg} / \mathrm{kg})+\operatorname{CsA}(5 \mathrm{mg} / \mathrm{kg})->41 \text { dias } \\
\text { TMC }(0.1 \mathrm{mg} / \mathrm{kg})+\operatorname{CsA}(5 \mathrm{mg} / \mathrm{kg})-103.8 \text { dias } \pm(56.8\end{array}$ \\
\hline Plesner et al. ${ }^{15}$ & $\mathrm{Balb} / \mathrm{c} \rightarrow \mathrm{EBA}$ & Sem imunossupressão & Cápsula renal & 60 dias \\
\hline Watanabe et al. ${ }^{16}$ & $\mathrm{Balb} / \mathrm{c} \rightarrow \mathrm{C} 57 \mathrm{BL} / 6$ & $\begin{array}{l}\text { Tacrolimus e DHMEQ (inibidor de } \\
\text { NF- kB) }\end{array}$ & Cápsula renal & 100 dias \\
\hline Gysemans et al. ${ }^{17}$ & $\mathrm{Balb} / \mathrm{c} \rightarrow \mathrm{C} 57 \mathrm{BL} / 6$ & Sem imunossupressão & Cápsula renal & $\begin{array}{l}9,2 \text { ( } \pm 4,9 \text { dias) (diabetes autoimune) } \\
15 \text { ( } \pm 3 \text { dias })\end{array}$ \\
\hline Xekouki et al. ${ }^{18}$ & Wistar $\rightarrow$ Lewis & CsA e MMF & Baço (parênquima) & $\begin{array}{l}8 \operatorname{dias}(\mathrm{Cs} A) \\
10.92 \operatorname{dias}(\text { MMF 1x) } \\
11 \operatorname{dias}(\text { MMF 2x) }\end{array}$ \\
\hline Baker et al. ${ }^{19}$ & $\mathrm{~A} / \mathrm{J} \rightarrow \mathrm{C} 57 \mathrm{Bl} / 6 \mathrm{~J}$ & Anticorpo monoclonal antiBIP-10 & Cápsula renal & $\begin{array}{l}19.7 \pm 2.3 \text { dias }(\mathrm{C} 57 \mathrm{Bl} / 6 \mathrm{~J}) \\
20.2 \pm 2.7 \text { dias }(\mathrm{CXCR} 3-/-\mathrm{C} 57 \mathrm{Bl} / 6 \mathrm{~J}\end{array}$ \\
\hline Li et al. ${ }^{20}$ & $\mathrm{FVB} \rightarrow \mathrm{Balb} / \mathrm{c}$ & Sem imunossupressão & Cápsula renal & N/A \\
\hline Vieiro et al..$^{21}$ & $\mathrm{C} 57 \mathrm{Bl} / 6 \rightarrow \mathrm{C} 3 \mathrm{H}$ & Timidina tritiada (pré-operatória) e CsA & Subcutâneo & $\mathrm{N} / \mathrm{A}$ \\
\hline Neuzillet et al. ${ }^{22}$ & $\mathrm{C} 3 \mathrm{H} \rightarrow \mathrm{Balb} / \mathrm{c}$ & Sem imunossupressão & Cápsula renal & 13.8 a 27.5 dias \\
\hline Melzi et al. ${ }^{23}$ & $\mathrm{C} 57 \mathrm{Bl} / 6 \rightarrow \mathrm{Balb} / \mathrm{c}$ & $\begin{array}{l}\text { Rapamicina+ FK506+ anti-IL- } \\
2 \mathrm{Ra}, \mathrm{mAbs} \text { e rapamicina+IL-10 }\end{array}$ & Cápsula renal & $>100$ dias \\
\hline Fiorina et al. ${ }^{24}$ & $\mathrm{Balb} / \mathrm{c} \rightarrow \mathrm{C} 57 \mathrm{Bl} / 6$ & Sem imunossupressão & Cápsula renal & 14 dias \\
\hline Fan et al. ${ }^{25}$ & $\mathrm{C} 57 \mathrm{Bl} / 6 \rightarrow \mathrm{Balb} / \mathrm{c}$ & $\begin{array}{l}\text { LTß R-Ig, CTLA4-Ig ou } \\
\text { LTRmAb antii-ratos }\end{array}$ & Cápsula renal & $\begin{array}{l}\text { LTß R-Ig - } 27 \text { dias, CTLA4-Ig - } 55 \text { dias, LTß } \\
\text { R-Ig+CTLA4-Ig->100 dias, LTRmAb anti rato- 11dias }\end{array}$ \\
\hline Jung et al. ${ }^{26}$ & $\mathrm{Balb} / \mathrm{c} \rightarrow \mathrm{C} 57 \mathrm{Bl} / 6$ & $\begin{array}{l}\text { CD154 mAb (MR1) anti-ratos e ROS-A } \\
\text { (Reactive Oxygen Specie- A) }\end{array}$ & Cápsula renal & $\begin{array}{l}\text { ROS-A - } 53 \text { dias, MR1 - } 82 \text { dias, ROS-A+MR1 - >160 } \\
\text { dias }\end{array}$ \\
\hline Påhlman et al. ${ }^{27}$ & $\mathrm{Balb} / \mathrm{c} \rightarrow \mathrm{C} 57 \mathrm{Bl} / 6 \mathrm{~J}$ & $\begin{array}{l}\text { AR-C117977 (10 ou } 30 \mathrm{mg} / \mathrm{kg}) \text { ou CsA } \\
20 \mathrm{mg} / \mathrm{kg}\end{array}$ & Cápsula renal & $\begin{array}{l}\text { CsA -16 dias AR-C117977, } 10 \mathrm{mg} / \mathrm{kg}->100 \text { dias } \\
\text { AR-C117977, } 30 \mathrm{mg} / \mathrm{kg}-29,33 \text { dias }\end{array}$ \\
\hline Wang et al. ${ }^{28}$ & $\mathrm{Balb} / \mathrm{c} \rightarrow \mathrm{C} 57 \mathrm{Bl} / 6$ & B7-H4 e Ad-LacZ & Cápsula renal & $\begin{array}{l}\text { B7-H4 - aproximadamente } 60 \text { dias Ad-LacZ - } \\
\text { aproximadamente }>20 \text { dias }\end{array}$ \\
\hline Chen et al. ${ }^{29}$ & $\begin{array}{l}\text { Sprague Dawley } \rightarrow \\
\text { Lewis }\end{array}$ & Sem imunossupressão & Intra-abdominal & 8 semanas \\
\hline Giraud et al..$^{30}$ & $\mathrm{C} 3 \mathrm{H} \rightarrow \mathrm{Balb} / \mathrm{c}$ & Sem imunossupressão & Cápsula renal & $\begin{array}{l}\text { SCOT + PEG } 20 \text { kDa } 10 \mathrm{~g} / \mathrm{L}-20 \text { dias, CMRL-1066 + } \\
1 \% \text { BSA - } 17.5 \pm 1 \text { dias, Solução UW - } 17.2 \pm 0.4 \text { dias, } \\
\text { SCOT sem PEG }-14 \pm 0.9 \text { dias } \\
\text { Solução HBSS }+0.5 \% \text { BSA- } 14 \pm 0.7 \text { dias }\end{array}$ \\
\hline Qi et al. ${ }^{31}$ & $\begin{array}{l}\text { Wistar/Lewis } \rightarrow \\
\text { Lewis }\end{array}$ & Sem imunossupressão & $\begin{array}{l}\text { Intraperitoneal } \\
\text { (macroencapsulados) } \\
\text { Cápsula renal (livre de } \\
\text { macroencapsulados) }\end{array}$ & $\begin{array}{l}24 \text { semanas (macroencapsulados) } 48 \mathrm{~h} \text { (livre de } \\
\text { macroencapsulados) }\end{array}$ \\
\hline Potiron et al. ${ }^{32}$ & Wistar $\rightarrow \mathrm{C} 57 \mathrm{Bl} / 6$ & CTLA4Ig ou CD40Ig & Cápsula renal & $24.3( \pm 9.7)$ dias \\
\hline Jahr et al. ${ }^{33}$ & Lewis $\rightarrow$ Wistar & Soro anti-rato antilinfócito & Fígado (veia porta) & N/A \\
\hline
\end{tabular}

$\mathrm{N} / \mathrm{A}=$ Não aferido 
Tabela 3: Análise de indução e tratamento do processo diabético com ilhotas transplantadas

\begin{tabular}{|c|c|c|c|c|c|c|}
\hline Autores & $\begin{array}{l}\text { Número } \\
\text { de ilhotas } \\
\text { transplantadas }\end{array}$ & $\begin{array}{l}\text { Método de indução } \\
\text { diabética }\end{array}$ & $\begin{array}{l}\text { Indução de } \\
\text { hiperglicemia } \\
\text { (pré-operatória) }\end{array}$ & $\begin{array}{l}\text { Normalização de } \\
\text { hiperglicemia } \\
\text { (pós-operatória) }\end{array}$ & Rejeição do enxerto & $\begin{array}{l}\text { Critérios para } \\
\text { disfunção primária } \\
\text { do enxerto (DPE) }\end{array}$ \\
\hline Fotiadis et al. ${ }^{9}$ & $1812( \pm 145)$ & $\begin{array}{l}\text { Estreptozotocina (60mg/ } \\
\mathrm{kg})+ \text { Solução PBS } \\
\text { (Phosphate Buffer } \\
\text { Solution) - 10mg/mL } \\
(\mathrm{pH} \mathrm{4,5);}\end{array}$ & 7 dias & 3 dias & 12 dias MMF e 10 dias CsA & $\begin{array}{l}\text { Glicose abaixo de } \\
200 \mathrm{mg} / \mathrm{dL} \text {; depois } \\
\text { do } 2 \text { PO } 2 \text { vezes } \\
\text { consecutivas }\end{array}$ \\
\hline Merani et al. ${ }^{10}$ & 1500 & $\begin{array}{l}\text { Estreptozotocina }(75 \mathrm{mg} / \\
\mathrm{kg} \text { ) intraperitoneal }\end{array}$ & 5 dias & 3 dias & 22 dias & $\begin{array}{l}\text { Glicose acima de } \\
324 \mathrm{mg} / \mathrm{dl} \text { depois de } \\
2 \text { dias consecutivos }\end{array}$ \\
\hline Nishimura et al. ${ }^{11}$ & $\begin{array}{l}\text { 2-10/câmara de } \\
\text { dobras cutâneas } \\
\text { dorsais }\end{array}$ & - & - & - & N/A & - \\
\hline Makhlouf et al. ${ }^{12}$ & $\begin{array}{l}350(\mathrm{Balb} / \mathrm{c}) \\
700(\mathrm{NOD})\end{array}$ & $\begin{array}{l}\text { Estreptozotocina e } \\
\text { espontaneamente }(225 \\
\mathrm{mg} / \mathrm{kg} \text { na cavidade } \\
\text { peritoneal) }\end{array}$ & 2 semanas & 3 dias & $\begin{array}{l}10 \text { dias (Balb/c) } \\
5 \text { dias (NOD) e } 7 \text { dias } \\
\text { rejeição completa (NOD) }\end{array}$ & $\begin{array}{l}200 \mathrm{mg} / \mathrm{dL}-2 \text { a } 3 \\
\text { dias consecutivos }\end{array}$ \\
\hline $\begin{array}{l}\text { Salazar-Bañuelos } \\
\text { et al. }{ }^{13}\end{array}$ & 840 (de Wistar) & - & - & - & N/A* & N/A \\
\hline Wee et al. ${ }^{14}$ & 4000 & $\begin{array}{l}\text { Estreptozotocina ( } 35 \\
\mathrm{mg} / \mathrm{kg})\end{array}$ & N/A & N/A & $\begin{array}{l}\text { Sem tratamento - } 5.2 \text { dias } \\
( \pm 0.5) \\
\text { TMC }-5,1 \text { dias }( \pm 0,9) \\
\text { TMC }(0.03 \mathrm{mg} / \mathrm{kg})+\mathrm{CsA} \\
(5 \mathrm{mg} / \mathrm{kg})->41 \mathrm{dias} \\
\mathrm{TMC}(0.1 \mathrm{mg} / \mathrm{kg})+\mathrm{CsA}(5 \\
\mathrm{mg} / \mathrm{kg})-103.8 \text { dias } \pm(56.8)\end{array}$ & $\begin{array}{l}200 \mathrm{mg} / \mathrm{dl} \text { após } 2 \\
\text { dias consecutivos }\end{array}$ \\
\hline Plesner et al. ${ }^{15}$ & 550 & $\begin{array}{l}\text { Estreptozotocina } \\
(375 \mathrm{mg} / \mathrm{dl}) \\
\text { intraperitoneal }\end{array}$ & $3-5$ dias & 5 dias* & 60 dias & $\begin{array}{l}\geq 198 \mathrm{mg} / \mathrm{dl} \text { após } 2 \\
\text { dias consecutivos }\end{array}$ \\
\hline Watanabe et al. ${ }^{16}$ & 600 ou 300 & $\begin{array}{l}\text { Estreptozotocina (180 } \\
\mathrm{mg} / \mathrm{kg} \text { ) intraperitoneal }\end{array}$ & $5-7$ dias & N/A & $\begin{array}{l}69 \text { dias (Tacrolimus) } \\
100 \text { dias (DHMEQ } 3 \text { dias e } \\
\text { Tacrolimus } 14 \text { dias) }\end{array}$ & $\begin{array}{l}>350 \mathrm{mg} / \mathrm{dL} \text { por } 2 \\
\text { dias consecutivos }\end{array}$ \\
\hline Gysemans et al. ${ }^{17}$ & 300 & Aloxano $(90 \mathrm{mg} / \mathrm{kg})$ & $24 \mathrm{~h}$ & N/A & N/A & $\begin{array}{l}\text { Glucose level > } \\
200 \mathrm{mg} / \mathrm{dl} \text { após } 3 \\
\text { dias consecutivos } \\
\text { ou mais }\end{array}$ \\
\hline Xekouki et al. ${ }^{18}$ & 2000 & $\begin{array}{l}\text { Estreptozotocina ( } 60 \mathrm{mg} / \\
\mathrm{kg} \text { ) diluída em solução } \\
\text { fosfatada } 10 \mathrm{mg} / \mathrm{mL}\end{array}$ & 1 semana & N/A & $7 \operatorname{dias}($ MMF 1x) & N/A \\
\hline Baker et al. ${ }^{19}$ & 300 & $\begin{array}{l}\text { Estreptozotocina } \\
(220 \mathrm{mg} / \mathrm{kg})\end{array}$ & N/A & N/A & 7 diass & N/A \\
\hline Li et al. ${ }^{20}$ & $\begin{array}{l}400 \text { (200/ } \\
\text { Cápsula renal) }\end{array}$ & $\begin{array}{l}\text { Estreptozotocina ( } 220 \\
\mathrm{mg} / \mathrm{kg})\end{array}$ & N/A & N/A & $\begin{array}{l}8.36 \pm 1.67 \text { (ilhotas de } \\
\text { FVB) } \\
16.2 \pm 2.52 \text { (ilhotas de } \\
\text { MT) }\end{array}$ & $\begin{array}{l}\text { Glicemia }> \\
250 \mathrm{mg} / \mathrm{dl} \text { por } \\
2 \text { medidas } \\
\text { consecutivas }\end{array}$ \\
\hline Vieiro et al. ${ }^{21}$ & 200 & $\begin{array}{l}\text { Estreptozotocina } \\
(270 \mathrm{mg} / \mathrm{kg}) \\
\text { intraperitoneal }\end{array}$ & N/A & N/A & 3-7 dias & $\begin{array}{l}\geq 250 \mathrm{mg} / \\
\mathrm{dL}-3 \text { medidas } \\
\text { consecutivas }\end{array}$ \\
\hline $\begin{array}{l}\text { Neuzillet et } \\
\text { al. }{ }^{22}\end{array}$ & 550 & N/A & $\mathrm{N} / \mathrm{A}$ & 4 horas & 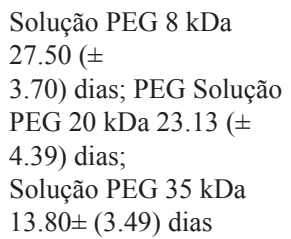 & $\begin{array}{l}>199.8 \mathrm{mmol} / \mathrm{L} \\
\text { por } 2 \text { medidas } \\
\text { consecutivas }\end{array}$ \\
\hline
\end{tabular}


Iuamoto LR, et al. Revisão sistemática sobre alotransplantes de ilhotas de Langerhans em roedores.

Tabela 3: Análise de indução e tratamento do processo diabético com ilhotas transplantadas

continuação

\begin{tabular}{|c|c|c|c|c|c|c|}
\hline Autores & $\begin{array}{l}\text { Número } \\
\text { de ilhotas } \\
\text { transplantadas }\end{array}$ & $\begin{array}{l}\text { Método de indução } \\
\text { diabética }\end{array}$ & $\begin{array}{l}\text { Indução de } \\
\text { hiperglicemia } \\
\text { (pré-operatória) }\end{array}$ & $\begin{array}{l}\text { Normalização de } \\
\text { hiperglicemia } \\
\text { (pós-operatória) }\end{array}$ & Rejeição do enxerto & $\begin{array}{l}\text { Critérios para } \\
\text { disfunção } \\
\text { primária do } \\
\text { enxerto (DPE) }\end{array}$ \\
\hline Melzi et al..$^{23}$ & 400 & $\begin{array}{l}\text { Estreptozotocina } \\
175 \text { a } 200 \mathrm{mg} / \mathrm{kg} \\
\text { intravenosa }\end{array}$ & $1-2$ semanas & 5 dias & $\begin{array}{l}29 \text { dias }(\text { ratos com } \\
\text { glicemeia }<450 \mathrm{mg} / \mathrm{dL}) \\
\text { e } 16 \text { dias }(\mathrm{ratos} \mathrm{com} \\
\text { glicemia }>450 \mathrm{mg} / \mathrm{dL})\end{array}$ & $\begin{array}{l}>250 \mathrm{mg} / \\
\mathrm{dL}-2 \text { medidas } \\
\text { consecutivas no } \\
\text { pós-operatório }\end{array}$ \\
\hline Fiorina et al. ${ }^{24}$ & N/A & Estreptozotocina & $\mathrm{N} / \mathrm{A}$ & N/A & 14 dias & $\mathrm{N} / \mathrm{A}$ \\
\hline Fan et al. ${ }^{25}$ & 500 & $\begin{array}{l}\text { Estreptozotocina ( } 200 \\
\mathrm{mg} / \mathrm{kg} \text { ) }\end{array}$ & N/A & N/A & $\begin{array}{l}27 \text { dias (LT[beta] R-Ig) } \\
55 \text { dias (CTLA4-Ig) } \\
\text { Depois de } 100 \text { dias ou } \\
\text { mais (LT[beta] R-Ig e } \\
\text { CTLA4-Ig) }\end{array}$ & $\begin{array}{l}>300 \mathrm{mg} / \\
\mathrm{d} 1-\text { após } 2 \text { dias } \\
\text { consecutivos }\end{array}$ \\
\hline Jung et al. ${ }^{26}$ & 300 IEQ & $\begin{array}{l}\text { Estreptozotocina (180 } \\
\mathrm{mg} / \mathrm{kg} \text { ) }\end{array}$ & N/A & 1 dia & $\begin{array}{l}\text { ROS-A - } 53 \text { dias } \\
\text { MR1 - } 82 \text { dias } \\
\text { ROS-A+MR1 - >160 } \\
\text { dias }\end{array}$ & $\begin{array}{l}>200 \mathrm{mg} / \\
\mathrm{d} \mathrm{-}-2 \text { medidas } \\
\text { consecutivas na } \\
\text { mesma semana }\end{array}$ \\
\hline Påhlman et al. ${ }^{27}$ & $500-600$ & Aloxano (intravenoso) & N/A & N/A & $\begin{array}{l}\text { CsA - } 16 \text { dias } \\
\text { AR-C117977, } 10 \mathrm{mg} / \\
\mathrm{kg}->100 \text { dias } \\
\text { AR-C117977, } 30 \mathrm{mg} / \mathrm{kg} \\
-29,33 \text { dias }\end{array}$ & $\mathrm{N} / \mathrm{A}$ \\
\hline Wang et al..$^{28}$ & 400 & $\begin{array}{l}\text { Estreptozotocina (200 } \\
\mathrm{mg} / \mathrm{kg} \text { ) }\end{array}$ & $3-4$ dias & 3 dias & $\begin{array}{l}\text { B7-H4 - } \\
\text { aproximadamente } 60 \\
\text { dias } \\
\text { Ad-LacZ - } \\
\text { aproximadamente > } \\
20 \text { dias }\end{array}$ & $\begin{array}{l}>250 \mathrm{mg} / \mathrm{dL} \text { após } \\
\text { o sucesso do } \\
\text { enxerto primário }\end{array}$ \\
\hline Chen et al. ${ }^{29}$ & 3000 IEQ & $\begin{array}{l}\text { Estreptozotocina } \\
\text { dissolvida em salina } \\
(50 \mathrm{mg} / \mathrm{kg})\end{array}$ & N/A & 1 semana & $\begin{array}{l}13 \text { semanas (SGA - } \\
\text { microencapsulados) } \\
7 \text { semanas (ABa- } \\
\text { microencapsulados) } \\
5 \text { semanas (APA- } \\
\text { microencapsulados) }\end{array}$ & N/A \\
\hline Giraud et al..$^{30}$ & 1400 IEQ & $\begin{array}{l}\text { Estreptozotocina } \\
(250 \mathrm{mg} / \mathrm{kg}) \\
\text { intraperitoneal }\end{array}$ & N/A & N/A & $\begin{array}{l}\text { SCOT + Solução PEG } \\
20 \text { kDa }{ }^{3} 10 \mathrm{~g} / \mathrm{L}-20 \\
\text { dias, } \\
\text { CMRL-1066 + 1\% } \\
\text { Solução BSA17.5 ( } \pm \\
\text { 1) dias, } \\
\text { Solução UW - } 17.2( \pm \\
0.4) \text { dias, } \\
\text { SCOT sem PEG -14 ( } \pm \\
0.9) \text { dias } \\
\text { HBSS + 0.5\% Solução } \\
\text { BSA - } 14( \pm 0.7) \text { dias }\end{array}$ & $\begin{array}{l}>200 \mathrm{mg} / \\
\mathrm{d} 1-2 \text { medidas } \\
\text { consecutivas }\end{array}$ \\
\hline Qi et al. ${ }^{31}$ & $1940( \pm 39)$ & $\begin{array}{l}\text { Estreptozotocina ( } 55 \\
\mathrm{mg} / \mathrm{kg} \text { ) }\end{array}$ & 1 semana & N/A & N/A & N/A \\
\hline Potiron et al..$^{32}$ & $800-1000$ & $\begin{array}{l}\text { Estreptozotocina (180 } \\
\mathrm{mg} / \mathrm{kg})\end{array}$ & 1 semana & 4 dias & $24.3( \pm 9.7)$ dias & $\begin{array}{l}250 \mathrm{mg} / \mathrm{dl} \\
\text { em } 2 \text { medidas } \\
\text { consecutivas }\end{array}$ \\
\hline Jahr et al. ${ }^{33}$ & $700-900$ & $\begin{array}{l}\text { Estreptozotocina ( } 55 \\
\mathrm{mg} / \mathrm{kg} \text { ) }\end{array}$ & $7-10$ dias & $\begin{array}{l}\text { Logo após } \\
\text { transplante }\end{array}$ & 1 semana & $\begin{array}{l}>300 \mathrm{mg} / \mathrm{dl} \text { após } \\
8.9( \pm 0.7) \text { dias }\end{array}$ \\
\hline
\end{tabular}

* Ratos que normalizaram os níveis de glicemia em 5 dias foram incluídos no estudo.

** A normoglicemia não foi atingida em recipientes com menos de 800 ilhotas transplantadas.

*** A normoglicemia foi mantida até o tempo de sacrifício (vários intervalos variando de 25-129 dias).

N/A = Não aferido.

IEQ $=$ Ilhotas Equivalentes 


\section{REFERÊNCIAS}

1. Chaves G. Incidência de diabetes tipo 1 aumenta $3 \%$ ao ano. Minas Gerais: Saúde Plena; 2013 [citado 21 jul. 2013]. Disponível em: http://goo.gl/FfCmxP.

2. Delfino VDA, Mocelin AJ. Transplante de pâncreas e de ilhotas pancreáticas: visão de nefrologista. Arq Bras Endocrinol Metab. 2002;46(2):177-85. http://dx.doi. org/10.1590/S0004-27302002000200011.

3. Sociedade Brasileira de Diabetes. São 12 milhões de diabéticos no Brasil. São Paulo; 2012 [citado 18 abr. 2012]. Disponível em: http://www.diabetes.org.br/sala-denoticias/2116-sao-12-milhoes-de-diabeticos-no-brasil.

4. Merani S, Shapiro AM. Current status of pancreatic islet transplantation. Clin Sci (Lond). 2006;110(6):611-25. doi: 10.1042/CS20050342.

5. United States of America. National Institute of Diabetes and Digestive and Kidney Diseases. Pancreatic islet transplantation. Bethesda, MD: NIH; 2007. (NIH Publication 07-4693). Available from: http://diabetes.niddk.nih.gov/dm/ pubs/pancreaticislet/Pancreatic Islet_Transplantation 508. pdf

6. Eliaschewitz DG, Franco DR, Mares-Guia TR, Noronha IL, Labriola L, Sogayar MC. Transplante de ilhotas na prática clínica: estado atual e perspectivas. Arq Bras Endocrinol Metab. 2009;53(1):15-23. http://dx.doi.org/10.1590/S000427302009000100004.

7. Shapiro AM, Lakey JR, Ryan EA, Korbutt GS, Toth E, Warnock GL, Kneteman NM, Rajotte RC. Islet transplantation in seven patients with type 1 diabetes mellitus using a glucocorticoid-free immunosuppressive regimen. N Engl J Med. 2000;343(4):230-8. doi: 10.1056/ NEJM200007273430401.

8. Ryan EA, Paty BW, Senior PA, Bigam D, Alfadhli E, Kneteman NM, Lakey JR, Shapiro AM. Five-year follow-up after clinical islet transplantation. Diabetes. 2005;54(7):2060-9. doi: 10.2337/diabetes.54.7.2060.

9. Fotiadis C, Xekouki P, Papalois AE, Antonakis PT, Sfiniadakis I, Flogeras D, Karampela E, Zografos G. Effects of mycophenolate mofetil vs cyclosporine administration on graft survival and function after islet allotransplantation in diabetic rats. World J Gastroenterol. 2005;11(18):2733-8. doi: 10.3748/wjg.v11.i18.2733.

10. Merani S, Pawlick RL, Edgar RL, Toso C, Emamaullee J, Anderson CC, Shapiro AM. Protein kinase C inhibitor, AEB071, acts complementarily with cyclosporine to prevent islet rejection in rats. Transplantation. 2009;87(1):59-65. doi: 10.1097/TP.0b013e31819198b4.

11. Nishimura R, Nishioka S, Fujisawa I, Shiku H, Shimada M, Sekiguchi S, Fujimori K, Ushiyama A, Matsue T, Ohuchi N, Satomi S, Goto M. Tacrolimus inhibits the revascularization of isolated pancreatic islets. PLoS One. 2013;8(4):1-8. doi: 10.1371/journal.pone.0056799.

12. Makhlouf L, Duvivier-Kali VF, Bonner-Weir S, Dieperink H, Weir GC, Sayegh MH. Importance of hyperglycemia on the primary function of allogeneic islet transplants. Transplantation. 2003;76(4):657-64. doi: 10.1097/01. ТР.0000080881.75767.0E

13. Salazar-Bañuelos A, Wright JR Jr, Sigalet D, BenítezBribiesca L. Pancreatic islet transplantation into the bone marrow of the rat. Am J Surg. 2008;195(5):674-8. doi: 10.1016/j.amjsurg.2007.12.040.

14. Wee YM, Choi MY, Kang CH, Kim YH, Kim JH, Lee SK, et al. The synergistic effect of Tautomycetin on Cyclosporine A-mediated immunosuppression in a rodent islet allograft model. Mol Med. 2010;16(7-8). Erratum in: Mol Med. 2012;18(1):321. doi: 10.2119/molmed.2009.00099.

15. Plesner A, Liston P, Tan R, Korneluk RG, Verchere CB. The $\mathrm{X}$-linked inhibitor of apoptosis protein enhances survival of murine islet allografts. Diabetes. 2005;54(9):2533-40. doi: 10.2337/diabetes.54.9.2533

16. Watanabe M, Yamashita K, Kamachi H, Kuraya D, Koshizuka Y, Shibasaki S, et al. Efficacy of DHMEQ, a NF$\kappa \mathrm{B}$ inhibitor, in islet transplantation: II. Induction DHMEQ treatment ameliorates subsequent alloimmune responses and permits long-term islet allograft acceptance. Transplantation. 2013;96(5):454-62. doi: 10.1097/TP.0b013e31829b077f.

17. Gysemans CA, Waer M, Valckx D, Laureys JM, Mihkalsky D, Bouillon R, Mathieu C. Early graft failure of xenogeneic islets in NOD mice is accompanied by high levels of interleukin-1. Diabetes. 2000;49(12):1992-7. doi: 10.2337/ diabetes.49.12.1992.

18. Xekouki P, Papalois A, Fotiadis C, Sfiniadakis J, Karampela E, Papadopoulou A, Grigoriou T, Sechas MN. In vivo test of two low doses of mycophenolate mofetil in an experimental model of islet allotransplantation. Transplant Proc. 2002;34(5):1446-8. doi:10.1016/S0041-1345(02)02922-6.

19. Baker MS, Chen X, Rotramel AR, Nelson JJ, Lu B, Gerard C, Kanwar Y, Kaufman DB. Genetic deletion of chemokine receptor CXCR3 or antibody blockade of its ligand IP10 modulates posttransplantation graft-site lymphocytic infiltrates and prolongs functional graft survival in pancreatic islet allograft recipients. Surgery. 2003;134(2):126-33. doi: 10.1067/msy.2003.213.

20. Li X, Chen H, Epstein PN. Metallothionein protects islets from hypoxia and extends islet graft survival by scavenging most kinds of reactive oxygen species. J Biol Chem. 2004;279(1):765-71. doi: 10.1074/jbc.M307907200.

21. Vieiro M, Ceballos C, Barrionuevo P, Hyon SH, Isturiz M, Argibay P. Inhibition of allospecific response in pancreatic islet transplantation: the glycan approach. Transplant Proc. 2005;37(1):242-4. doi:10.1016/j.transproceed.2004.12.298

22. Neuzillet Y, Giraud S, Lagorce L, Eugene M, Debre P, Richard F, Barrou B. Effects of the molecular weight of peg molecules $(8,20$ and $35 \mathrm{KDA})$ on cell function and allograft survival prolongation in pancreatic islets transplantation. Transplant Proc. 2006;38(7):2354-5. doi: 10.1016/j. transproceed.2006.06.117.

23. Melzi R, Battaglia M, Draghici E, Bonifacio E, Piemonti L. Relevance of hyperglycemia on the timing of functional 
loss of allogeneic islet transplants: implication for mouse model. Transplantation. 2007;83(2):167-73. doi: 10.1097/01. tp.0000250659.24286.43.

24. Fiorina P, Jurewicz M, Tanaka K, Behazin N, Augello A, Vergani A, von Andrian UH, Smith NR, Sayegh MH, Abdi $\mathrm{R}$. Characterization of donor dendritic cells and enhancement of dendritic cell efflux with CC-chemokine ligand 21: a novel strategy to prolong islet allograft survival. Diabetes. 2007;56(4):912-20. doi: 10.2337/db06-144.

25. Fan K, Wang H, Wei H, Zhou Q, Kou G, Hou S, Qian W, Dai J, Li B, Zhang Y, Zhu T, Guo Y. Blockade of LIGHT/ HVEM and B7/CD28 signaling facilitates long-term islet graft survival with development of allospecific tolerance. Transplantation. 2007;84(6):746-54. doi: 10.1097/01. tp.0000280545.14489.df.

26. Jung da Y, Kim EY, Joo SY, Park JB, Moon C, Kim SH, Sim EY, Joh JW, Kwon CH, Kwon GY, Kim SJ. Prolonged survival of islet allografts in mice treated with rosmarinic acid and anti-CD154 antibody. Exp Mol Med. 2008;40(1):110. doi:10.3858/emm.2008.40.1.1.

27. Påhlman C, Malm H, Qi Z, Veress B, Ferguson D, Bundick R, Murray C, Donald D, Ekberg H. Operational tolerance in nonvascularized transplant models induced by AR-C117977, a monocarboxylate transporter inhibitor. Transplantation. 2008;86(8):1135-8. doi: 10.1097/TP.0b013e318186b978.

28. Wang X, Hao J, Metzger DL, Mui A, Ao Z, Verchere CB, Chen L, Ou D, Warnock GL. Local expression of B7-H4 by recombinant adenovirus transduction in mouse islets prolongs allograft survival. Transplantation. 2009;87(4):48290. doi: 10.1097/TP.0b013e318195e5fa.

29. Chen X, Shao W, Chen JB, Zhang L, Matthias C, Shan SG, Qi ZQ. Allotransplantation of sulphate glucomannan-alginate barium (SGA)-microencapsulated rat islets for the treatment of diabetes mellitus. Immunol Invest. 2009;38(7):561-571. doi: 10.1080/08820130902984828.

30. Giraud S, Bon D, Neuzillet Y, Thuillier R, Eugene M, Hauet T, Barrou B. Concentration and chain length of polyethylene glycol in islet isolation solution: evaluation in a pancreatic islet transplantation model. Cell Transplant. 2012;21(9):2079-88. doi: 10.3727/096368912X638928.

31. Qi Z, Yamamoto C, Imori N, Kinukawa A, Yang KC, Yanai G, Ikenoue E, Shen Y, Shirouzu Y, Hiura A, Inoue $\mathrm{K}$, Sumi S. Immunoisolation effect of polyvinyl alcohol (PVA) macroencapsulated islets in type 1 diabetes therapy. Cell Transplant. 2012;21(2-3):525-34. doi: 10.3727/096368911X605448.

32. Potiron N, Chagneau C, Boeffard F, Soulillou JP, Anegon I, Le Mauff B. Adenovirus-mediated CTLA4Ig or CD40Ig gene transfer delays pancreatic islet rejection in a rat-tomouse xenotransplantation model after systemic but not local expression. Cell Transplant. 2005;14(5):263-75. http:// dx.doi.org/10.3727/000000005783983052.

33. Jahr H, Hussmann B, Eckhardt T, Bretzel RG. Successful single donor islet allotransplantation in the streptozotocin diabetes rat model. Cell Transplant. 2002;11(6):513-8. http:// dx.doi.org/10.3727/000000002783985576. 